\title{
Gamma-ray generation in ultrahigh-intensity laser-foil interactions
}

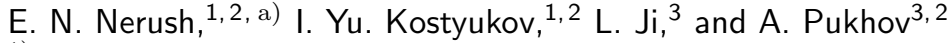 \\ ${ }^{1)}$ Institute of Applied Physics of the Russian Academy of Sciences, 46 Ulyanov St., 603950 Nizhny Novgorod, \\ Russia \\ ${ }^{2)}$ University of Nizhny Novgorod, 23 Gagarin Avenue, Nizhny Novgorod 603950, \\ Russia \\ ${ }^{3)}$ Heinrich-Heine-Universitat Dusseldorf, 40225 Dusseldorf, Germany
}

(Dated: 31 October 2018)

Incoherent photon emission by ultrarelativistic electrons in the normal incidence of a laser pulse on a foil is investigated by means of three-dimensional numerical simulations in the range of intensities $2 \times 10^{21}-2 \times$ $10^{25} \mathrm{~W} \mathrm{~cm}^{-2}$ and electron densities $2 \times 10^{22}-1 \times 10^{24} \mathrm{~cm}^{-3}$. We focus on properties of the resulting synchrotron radiation, such as its overall energy, directivity of the radiation pattern and slope of the energy spectrum. Regimes of laser-foil interactions are studied in the framework of a simple analytical model. The laser-plasma parameters for efficient gamma-ray generation are found and revealed to be close to the parameters for relativistic foil motion. It is shown that in the case of oblique incidence of a $3 \mathrm{PW}, 10 \mathrm{fs}$ laser pulse on a thin foil about $10^{8}$ photons $/ 0.1 \%$ bandwidth are produced at the energy level of $1 \mathrm{MeV}$ that significantly exceeds performance of the modern Compton gamma-ray sources. Various applications of the gamma-ray bunches are discussed.

\section{INTRODUCTION}

Nowadays gamma-ray sources are widely used in numerous applications from medicine to nuclear physics. Such sources can be based not only on the radioactivity, bremsstrahlung ${ }^{1}$ and synchrotron emission in magnetic field ${ }^{2}$ but also on a Compton scattering of laser light on electron beams of conventional accelerators $^{3-5}$. The recent progress in laser technologies and laser-plasma electron acceleration opens the opportunity to eliminate a conventional electron accelerator from the scheme and develop a compact all-optical Compton source of hard photons ${ }^{6}$ or a laser-plasma synchrotron source ${ }^{7}$. Laser-produced hot electrons can also generate bremsstrahlung photons ${ }^{8}$, however, the efficiency of this generation mechanism is not high due to a low value of the bremsstrahlung cross-section. Nevertheless, the abundant positron production via decay of bremsstrahlung photons in thick high-Z targets is demonstrated ${ }^{9}$.

Another mechanism of gamma-ray production is the nonlinear Compton scattering induced directly by laserdriven plasma electrons. For a laser field of ultrarelativistic intensity the nonlinear Compton scattering is equivalent to synchrotron radiation ${ }^{10}$, i.e. ultrarelativistic plasma electrons emit gamma-rays during travelling along curved trajectories. The resulting radiation losses take away a considerable part of the electron energy and significantly affect electron motion if the laser intensity becomes greater than $10^{23} \mathrm{~W} \mathrm{~cm}^{-2}$ (for optical wavelengths), the intensity of the so-called radiationdominated regime ${ }^{11}$.

Recent results on the generation of laser pulses with high intensity (up to $2 \times 10^{22} \mathrm{~W} \mathrm{~cm}^{-2}$, see Ref. 12) and a

\footnotetext{
a) Electronic mail: nerush@appl.sci-nnov.ru
}

number of proposals for multipetawatt and exawatt laser facilities ${ }^{13}$ stimulate theoretical study of laser-plasma synchrotron sources. E.g., generation of gamma-ray bunches in the interaction of a laser pulse with a tailored overcritical density target is investigated on the basis of theoretical analysis and 2D particle-in-cell simulations with the radiation friction force incorporated 14 . The theoretical analysis is done for a circularly polarized laser pulse propagating in an underdense plasma. The obtained analytical results agrees qualitatively with the results of simulations for linear polarization. More complex plasma dynamics than it is assumed in Ref. 14 is revealed in Ref. 15 for an intense laser pulse interacting with a relativistically underdense plasma. The $2 \mathrm{D}$ simulation shows that a great portion of plasma electrons moves towards the laser pulse during a noticeable part of an optical cycle. At this time interval the intensity of the synchrotron radiation emitted by the electrons peaks, that leads to generation of a $90^{\circ}$-wide gamma-ray radiation pattern directed towards the laser pulse. Such backand-forth electron motion (including the case of opaque plasmas) is a key element of the high-harmonic generation theory ${ }^{16}$, where a coherent part of the synchrotron radiation from laser-irradiated solids is investigated. It is interesting to note that in Ref. 17 a $2 \mathrm{D}$ simulation of a laser pulse interacting with a relativistically opaque plasma reveals mostly forward-directed gamma-ray radiation pattern.

Laser-plasma interactions potentially become even more complicated at the intensity of $10^{24} \mathrm{~W} \mathrm{~cm}^{-2}$, when avalanche-like generation of gamma-quanta and electronpositron $\left(e^{+} e^{-}\right)$pairs can occur ${ }^{18,19}$ due to consecutive events of nonlinear Compton scattering and BreitWheeler process. The produced $e^{+} e^{-}$plasma can noticeably affect the interaction process ${ }^{20}$ and can even cause a significant absorption of the laser field ${ }^{19,21}$. In the latter case a sizeable portion of the initial laser energy is converted into the energy of $\mathrm{MeV}$ photons (30\% in the 
simulation of Ref. 21) which can have anisotropic distribution in some cases 22 .

In this paper we attempt to classify gamma-ray generation regimes and examine the influence of plasma dynamics, ion acceleration and other effects on the gamma-ray generation process. For this purpose we perform a series of numerical simulations for a wide range of foil densities and laser intensities, as described in Sec. III. Despite of obvious limitations of such a consideration (e.g., we restrict the simulations to some certain value of the foil thickness), it allows us to determine the region of the most efficient gamma-ray generation (see Sec. III).

At ultrahigh intensities any light nucleus become fully ionized, hence the ion charge-to-mass ratio doesn't depend much on the material type. Thus, the presented picture of laser-foil interactions doesn't depend much on the material type. Electron dynamics, generation of electromagnetic fields and ion acceleration are considered in Sec. III and Sec. IV Though a plenty of important effects manifest itself in the considered parameter region, it occurs that gamma-ray generation is strongly affected by ion motion, and the region of efficient gamma-ray generation approximately coincides with the region of relativistic ion dynamics (see Sec. IV).

Having in mind possible applications of $\mathrm{MeV}$ photons, we focus on gamma-ray angular distribution and spectrum along with gamma-ray generation efficiency. Characteristics of gamma-ray bunches from laser-irradiated foils obtained by means of simulations in a wide range of parameters are discussed in Sec. III and Sec. V. In Sec. $\nabla$ two sets of laser-plasma interaction parameters and the parameters of the resulting gamma-ray sources are considered in detail. Namely, we discuss the gammaray generation with normally incident $100 \mathrm{PW}$ laser pulse and with obliquely incident $3 \mathrm{PW}$ laser pulse. In the first case high laser-to-gamma-ray conversion efficiency (9\%) can be easily got. In the second case tight focusing of the laser pulse and an accurate choice of the plasma density and the incidence angle let obtain reasonable conversion efficiency $(\sim 1 \%)$ along with quite high directivity of a single-lobe radiation pattern. Possible applications of the corresponding gamma-ray bunches are discussed and the comparison with the existing gamma-ray sources is also given.

\section{MAP OF THE SOURCE GAIN}

Three-dimensional numerical simulations allow us to calculate gamma-ray radiation pattern, i.e. the directional (angular) dependence of the emitted energy integrated in time. We introduce the directivity $\mathcal{D}$ of the source as the ratio of the radiation pattern maximum to its value in the case of isotropic distribution of the emitted gamma-rays. Since high directivity of a gammaray beam is more reasonable for applications, here we focus on the gain $\mathcal{G}$ that is the product of the directivity and the generation efficiency $\mathcal{E}$, where we introduce $\mathcal{E}$

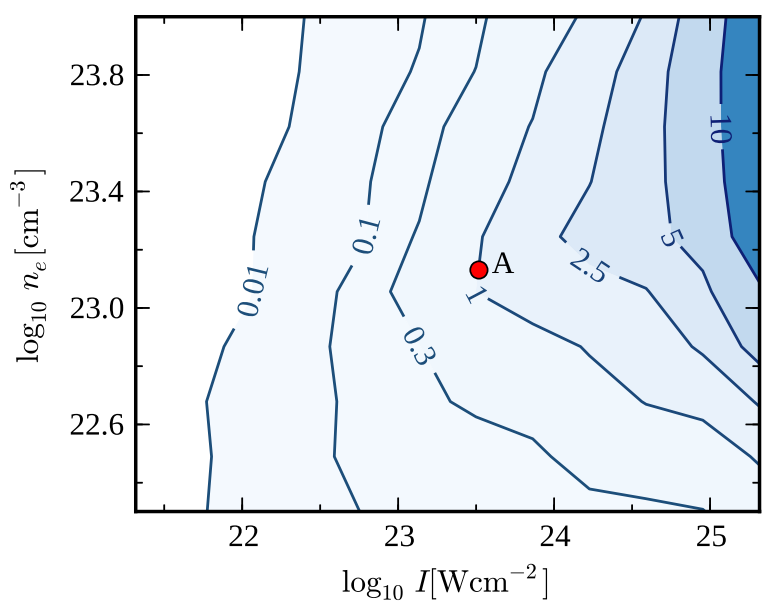

FIG. 1. The gain $\mathcal{G}$, i.e. the product of the radiation pattern directivity and the gamma-ray generation efficiency, for highenergy photons generated at the normal incidence of a $10 \mathrm{fs}$ linearly polarized laser pulse on a $0.91 \mu \mathrm{m}$ thick plasma slab (see text for further details).

as the overall energy of the gamma-rays divided by the initial energy of the laser pulse. The distribution of the gain on the laser-plasma interaction parameters is shown in Fig. 1, where interpolation based on 120 simulation runs is presented. Every computed value corresponds to the normal incidence of a linearly polarized laser pulse on a fully ionized thin foil. The normalized amplitude of a laser pulse $a_{0}=e E_{0} / m c \omega$ alters from 25 to 2500 , and the initial plasma density normalized to the critical density $n_{0}=n_{e} / n_{c r}$ lies in the range $15-745$, where $n_{c r}=m \omega^{2} / 4 \pi e^{2}, e>0$ and $m$ are the magnitude of the electron charge and the electron mass, respectively, $\omega=2 \pi c / \lambda, \lambda=0.91 \mu \mathrm{m}$ is the laser wavelength and $c$ is the speed of light. The laser pulse has Gaussian envelope with duration 10 fs and width $5.4 \mu \mathrm{m}$ (both are measured as FWHM of the laser intensity), the initial distance between irradiated foil boundary and the laser pulse centre is $5 \lambda$, the interaction time is $12 \lambda / c$, the foil thickness is $l=1 \lambda$, the ion mass-to-charge ratio is $M / Z=2$, where $M$ is the ion mass normalized to the proton mass and $Z$ is the ion charge normalized to $e$.

The simulations are performed with a particle-in-cell (PIC) code that takes into account incoherent emission and decay of hard photons using the Monte Carlo (MC) technique. The separation of the electromagnetic fields on a low-frequency (laser field and its harmonics) and high-frequency (gamma-rays) parts is possible due to a wide gap between them: spectrum of coherent harmonics emitted in laser-solid interactions typically extends up to $1 \mathrm{keV} \stackrel{16}{ }$, and the characteristic energy of incoherent photons produced by a laser pulse striking a solid is greater or of the order of $1 \mathrm{MeV}$ if laser intensity is greater than $10^{22} \mathrm{~W} \mathrm{~cm}^{-2}$ (for optical wavelengths).

The PIC part of the code that describes laser and plasma fields and plasma dynamics is based on the same 


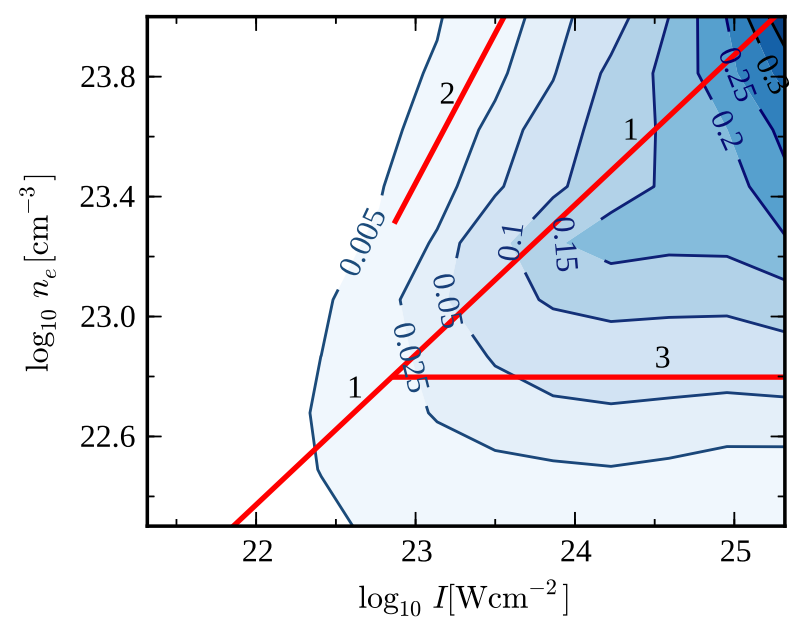

FIG. 2. The gamma-ray generation efficiency $\mathcal{E}$ in laser-foil interaction obtained in the same simulation series as used for Fig. 1 Lines 1-3 show distinctive interaction regimes. Below line 1 electrons and ions are easily separated by the laser field and the laser pulse propagates through the plasma slab, above this line the plasma reflects the laser pulse. The region bounded by lines $2-3$ corresponds to quick foil acceleration up to relativistic velocity (see Sec. IV).

methods as the code VLPL ${ }^{23}$, however, we use the Vay's particle pusher ${ }^{24}$ instead of Boris pusher. This part of the code has been used for a study of various plasma problems, for example, for simulations of laser-plasma electron acceleration 25 . The effects of quantum electrodynamics (QED), namely emission of hard photons and hard photon decay are simulated by an alternative event generator ${ }^{26}$ that uses the Baier-Katkov formulae for the event probabilities 27,28 which are applicable in quantum limit (when photon recoil is substantial) as well as in classical limit. The MC part of the code has been used for numerical simulations of electromagnetic cascades in a laser field ${ }^{21,26}$.

It follows from the simulations that gamma-ray generation is strongly affected by ion dynamics. The gammaray generation efficiency is shown in Fig. 2, as well as the boundaries of the characteristic laser-foil interaction regimes. The ions are quickly accelerated up to relativistic velocities within the region bounded by lines 2 and 3 , that fairly well coincides with the region of the sizeable gamma-ray generation efficiency. The electron dynamics in laser-foil interactions is discussed in Sec. III and analytical estimations that lead to lines 2 and 3 in Fig. 2 are considered in Sec. IV]

\section{ELECTRON MOTION IN LASER-SOLID INTERACTIONS}

Electron dynamics in laser-foil interactions can be extremely diverse and complicated. Nonetheless, as a rule, plasmas prevent laser pulse penetration and a thin and dense electron layer which screens the incident field is formed at the front of the laser pulse. As we show below, this property together with one-dimensional approximation is enough for a rough description of the electron trajectories.

Dynamics of the electron layer that screens the incident field is extensively discussed in the context of highharmonic generation in laser-solid interactions $\frac{16}{}$. It is shown that at some specific interaction parameters relativistic electron layers (bunches) with nanometer thickness and density up to four orders of magnitude higher than nonrelativistic critical plasma density can be formed and coherently emit high laser harmonics (up to the photon energy of about $1 \mathrm{keV}$ that corresponds to the wavelengths of $1 \mathrm{~nm}$ ). At the same time a thicker electron layer, which doesn't emit high harmonics efficiently but also screens incident field and prevents penetration of the laser pulse into the plasma, is formed in a very broad range of the interaction parameters. Despite such a layer doesn't emit keV photons coherently, electrons in the layer can produce $\mathrm{MeV}$ photons due to incoherent synchrotron emission.

For the description of the electron trajectories we assume that (i) for normal incidence of a laser pulse on a plasma halfspace in the framework of one-dimensional geometry a flat thin (much thinner than laser wavelength) electron layer is formed that (ii) fully compensates incident laser field behind the layer by layer's fields, hence, the plasma behind the layer is unperturbed. Additionally we assume that (iii) ions remain immobile during the interaction and (iv) initial plasma density is restored behind the layer if it moves towards the laser pulse. Hence, the surface charge density of the layer is $n_{0} x_{l}$, where $x_{l}$ is the distance between the initial position of the plasma boundary and the layer, normalized to $c / \omega$.

The coherent part of the electromagnetic radiation emitted by the layer at a particular time instance can be easily found from Maxwell's equations in the reference frame $K^{\prime}$ where the layer moves along itself:

$$
E_{\rightarrow}^{\prime}=B_{\rightarrow}^{\prime}=E_{\leftarrow}^{\prime}=-B_{\leftarrow}^{\prime}=-J^{\prime} / 2,
$$

where $E$ and $B$ denote the fields of the emitted waves, $y$ component of the electric field and $z$ component of the magnetic field, respectively, fields are converted from CGS electrostatic units to the units of $m c \omega / e$, the axes of the $K^{\prime}$ reference frame are assumed to be parallel to the axes of the laboratory frame of reference $K$ in which the linearly polarized laser pulse is incident along the $x$ axis and the electric field of the pulse directed along the $y$ axis. The symbols " $\rightarrow$ " and " $\leftarrow$ " denote the fields of the waves running in $+x$ and $-x$ directions at the corresponding layer boundaries, respectively,

$$
J^{\prime}=\int_{x_{l}^{\prime}-\delta x_{l}^{\prime} / 2}^{x_{l}^{\prime}+\delta x_{l}^{\prime} / 2} j^{\prime}\left(x^{\prime}\right) d x^{\prime}
$$

is the layer surface current density in $K^{\prime}, j$ is the volume current density in the units of $m c \omega^{2} / 4 \pi e, x_{l}^{\prime}+\delta x_{l}^{\prime} / 2$ and 
$x_{l}^{\prime}-\delta x_{l}^{\prime} / 2$ are the coordinates in $K^{\prime}$ of the layer boundaries normalized to $c / \omega$, hence, $\delta x_{l}^{\prime}$ is the layer thickness. Lorentz transformation of the coherently emitted fields yields for the laboratory reference frame:

$$
\begin{gathered}
E_{\rightarrow}=B_{\rightarrow}=-\frac{J}{2\left(1-v_{x}\right)}, \\
E_{\leftarrow}=-B_{\leftarrow}=-\frac{J}{2\left(1+v_{x}\right)},
\end{gathered}
$$

where

$$
J=-n_{0} x_{l} v_{y}
$$

$v_{x} \approx d x_{l} / d t$ and $v_{y}$ are the components of the speed of electrons that form the layer, $x_{l}$ is the distance between the layer and the initial (unperturbed) position of the irradiated plasma boundary in the reference frame $K, t$ is the current time instance normalized to $\omega^{-1}$, and $n_{0}$ is the initial plasma density normalized to $n_{c r}=$ $m \omega^{2} / 4 \pi e^{2}$. We note again that $n_{0} x_{l}$ is the surface charge density of the electron layer.

The layer motion can be found now from the assumption that the plasma remains unperturbed behind the layer because in this region the incident wave [with $\left.E_{y}(x, t)=\tilde{E}(x-t), \tilde{E}_{z}(x, t)=0\right]$ is fully compensated by the wave emitted by the electron layer:

$$
\tilde{E}\left(x_{l}-t\right)+E_{\rightarrow}=0 .
$$

Assuming that particles in the layer are ultrarelativistic and $v_{x}^{2}+v_{y}^{2} \approx 1$, the latter equation can be rewritten as follows:

$$
\frac{d x_{l}}{d t}=\frac{4 \tilde{E}^{2}\left(x_{l}-t\right)-n_{0}^{2} x_{l}^{2}}{4 \tilde{E}^{2}\left(x_{l}-t\right)+n_{0}^{2} x_{l}^{2}} .
$$

Electrons can leave and join the layer during its motion, nevertheless, we use Eq. (7) in order to determine some average trajectory as follows. As $x_{l}(t)$ is found from Eq. (7), then $v_{x}(t)$ and $v_{y}= \pm \sqrt{1-v_{x}^{2}}$ can be obtained, and sign of $v_{y}$ is chosen to satisfy Eq. (6).

Eq. (77) is not based on the equations of electron motion, and it should not be affected much by such effects as radiation losses and back reaction of the coherently emitted fields on the layer. Hence, the main limitation of this equation is the assumption of immobile ions.

Due to relativistic motion of the layer $E_{\rightarrow} \neq E_{\leftarrow}$, hence, the amplitude and the shape of the reflected laser light can be considerably modified in comparison with the incident light. This also means that the fields of the incident laser pulse are not compensated inside the electron layer. However, the Lorentz factor of the layer electrons cannot be estimated in the framework of the proposed model because the model leads to the following:

$$
\frac{d \gamma}{d t}=-\mathbf{v E} \approx-v_{x} E_{x}-v_{y}\left(\tilde{E}+E_{\leftarrow}\right)=0,
$$

where we assume that $E_{x}=n_{0} x_{l}$. Hence, energy gain of the electrons is caused by more subtle effects such as

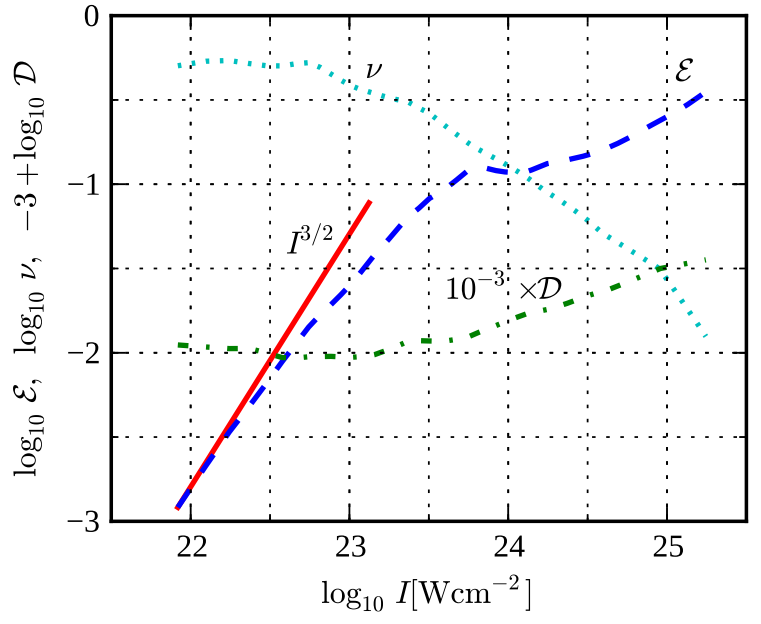

FIG. 3. (Dashed line) The gamma-ray generation efficiency $\mathcal{E}$, (dotted line) the spectral coefficient $\nu$, (dashed-dotted line) the directivity $\mathcal{D}$ at fixed value of the similarity parameter $S=2 / l=1 / \pi$ (i.e., along line 1 in Figs. 2 and 4) and (solid line) the curve $\mathcal{E} \propto I^{3 / 2}$ versus laser intensity. Results of numerical simulations for the same interaction parameters as used for Fig. 1

finiteness of the gamma-factor, dispersion of electron parameters, electron inertia, ion motion etc. that hardly could be taken into account. Since electron gamma-factor is the crucial parameter for incoherent photon emission, gamma-ray generation cannot be described only on the basis of Eq. (7). Nevertheless, Eq. (7) allows us to estimate the depth on that laser pulse pushes the electron layer.

Maximal value of the layer displacement $X_{l}$ corresponds to $d x_{l} / d t=0$. Assuming that the maximal displacement corresponds also to the maximal value of the incident field $\tilde{E} \approx a_{0}$, we obtain:

$$
X_{l} \simeq \frac{2}{S}, \quad S=\frac{n_{0}}{a_{0}},
$$

where $S$ is the so-called similarity parameter ${ }^{29}$. Eq. (9) is very useful for a qualitative description of the ion dynamics, as shown in the next section. Moreover, Eqs. (7) and (9) can be used in order to analyse gamma-ray parameters as follows.

For the maximal number of electrons in the layer Eq. (9) yields $N \propto n_{0} X_{l} \sim a_{0}$. It also follows from the considered model that the shape of the electron trajectories, hence, the curvature radius, depends only on the similarity parameter $S$. Since synchrotron emitting power proportional to $\gamma^{4}$ for the fixed curvature radius, for fixed $S$ the gamma-ray generation efficiency scales as $\mathcal{E} \propto N \gamma^{4} / I \propto I^{3 / 2}$ under the assumption that the average electron Lorentz factor $\gamma \sim a_{0}$. The dependence of the gamma-ray generation efficiency on laser intensity at fixed $S$ is shown in Fig. 3 along with the fit $\mathcal{E} \propto I^{3 / 2}$ that fairly well coincides with numerical data at low intensities. 


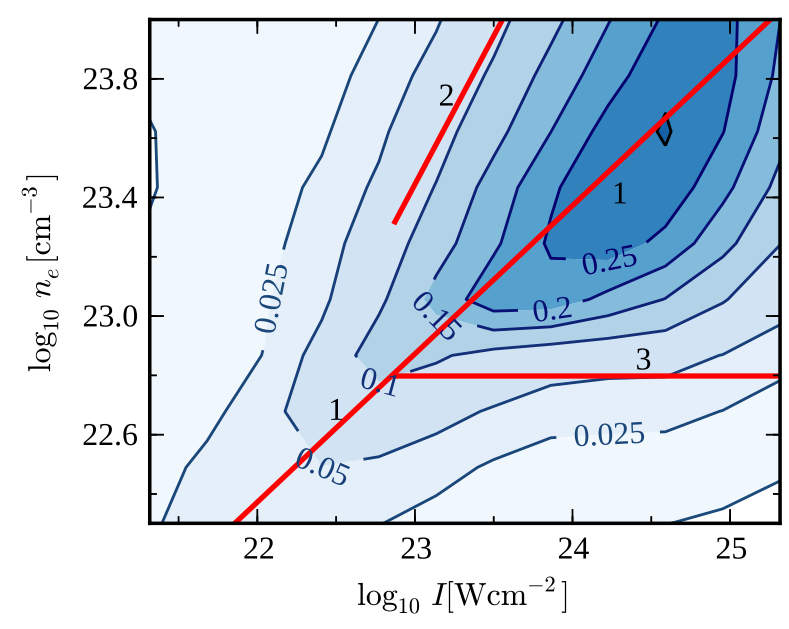

FIG. 4. The overall ion energy normalized to the initial energy of the laser pulse for the same set-up as in Fig. 1. Lines 1, 2 and 3 are the same as in Fig. 2.

The dependence of the directivity $\mathcal{D}$ and spectral coefficient $\nu$ on intensity is also shown in Fig. 3. The coefficient $\nu$ is determined by the root-mean-square fitting in the logarithmic axes of the obtained gamma-ray spectrum by the classical synchrotron spectrum of a single electron 30 :

$$
\begin{gathered}
\frac{d N_{p h}}{d \omega_{p h}} \propto \int_{\kappa}^{\infty} \operatorname{Ai}(\xi) d \xi+\frac{2}{\kappa} \operatorname{Ai}^{\prime}(\kappa) \\
\kappa=\left(\frac{\omega_{p h}}{\nu a_{0}^{3} \omega}\right)^{2 / 3}
\end{gathered}
$$

where $\hbar \omega_{p h}$ is hard-photon energy. Actually in the classical synchrotron spectrum $\kappa^{3 / 2}=\omega_{p h} / F_{\perp} \gamma^{2} \omega$, where $F_{\perp}$ is the perpendicular to the electron velocity component of the force that causes photon emission, normalized to $m c \omega$. Obviously, we set $F_{\perp} \gamma^{2}=\nu a_{0}^{3}$, hence, $\nu$ can characterize such effects as decrease of the electron Lorentz factor and decrease of the angle between $\mathbf{F}$ and the electron velocity caused by the radiation reaction 19 . We found that if the appropriate spectral coefficient $\nu$ is chosen, Eq. (10) describes well enough the part of the gamma-ray spectrum extended from the gamma-ray energy cut-off to 0.2 of its value.

It should be noted that for $I \lesssim 10^{23} \mathrm{~W} \mathrm{~cm}^{-2}$ the directivity and the spectral coefficient do not change with the intensity (see Fig. 3), that conforms to the proposed analytical model. Besides, for $I \gtrsim 10^{23} \mathrm{~W} \mathrm{~cm}^{-2}, \nu$ declines apparently due to radiation losses and $\mathcal{D}$ increases with the increase of the intensity. The latter can be interpreted as the radiation pattern narrowing due to the light aberration and the relativistic Doppler effect which are caused in turn by relativistic motion of the entire foil. This effect is also considered in Ref. 17.

\section{ION DYNAMICS IN LASER-FOIL INTERACTIONS}

Regimes of laser-foil interactions in terms of ion motion can be classified by means of Eq. (9). First, the line

$$
S=\frac{2}{l},
$$

where $l$ is the foil thickness, obviously, corresponds to a complete separation of the electrons and the ions at the maximal magnitude of the laser intensity. Under the assumption of immobile ions, for $S>2 / l$ the rear side of the foil remains unperturbed, otherwise, for $S<2 / l$, almost all electrons are expelled from the foil. Eq. (12) corresponds to line 1 in Figs. 2 and 4.

The magnitude of the electric field that accelerates the ions for $S>2 / l$ can be estimated as follows:

$$
E_{x} \simeq n_{0} X_{l}=2 a_{0}
$$

Let us introduce the time interval $\tau$ such that ions are accelerated by $E_{x}$ up to relativistic velocities during this interval. Hence, $2 \tau a_{0, i} \approx 1$, where

$$
a_{0, i}=\frac{m}{m_{p}} \frac{Z}{M} a_{0} .
$$

Here $m_{p} \approx 1836 m$ is the proton mass. At the same time, relativistic ions pass the distance $X_{l}$ during the time $X_{l}$, equating that to $\tau$ yields the following relation between $a_{0}$ and $n_{0}$ :

$$
n_{0} \simeq 4 a_{0} a_{0, i}
$$

Thus, if the plasma density is higher than that given by Eq. (15), $X_{l}$ is small and the ions have time to leave the accelerating gap before they gain relativistic velocities. In the opposite case, if the plasma density is lower than the threshold Eq. (15), accelerating gap is thick enough and the ions at the front of the foil become relativistic. In the latter case the foil is crumpled and blown away by the laser pulse with relativistic velocity until the density of the shovelled plasma is not high enough to slow down the process. Eq. (15) corresponds to line 2 in Figs. 2 and 4. It is worth to note that the estimations derived above relates mostly to high enough laser intensities $\left(a_{0, i} \gtrsim\right.$ $1 / 4 \pi)$. Otherwise $\tau$ becomes greater than the laser period and the ion acceleration process depends on the laser pulse duration.

The next threshold that corresponds to relativistic ion dynamics can be found in the region $S<2 / l$. The completely separated electrons and ions generate in this case the following accelerating field:

$$
E_{x} \simeq n_{0} l
$$

This field accelerates the ions up to relativistic velocities during the laser period if $n_{0}>\hat{n}_{0}$, where

$$
\hat{n}_{0}=\frac{1}{2 \pi l} \frac{m_{p}}{m} \frac{M}{Z} .
$$




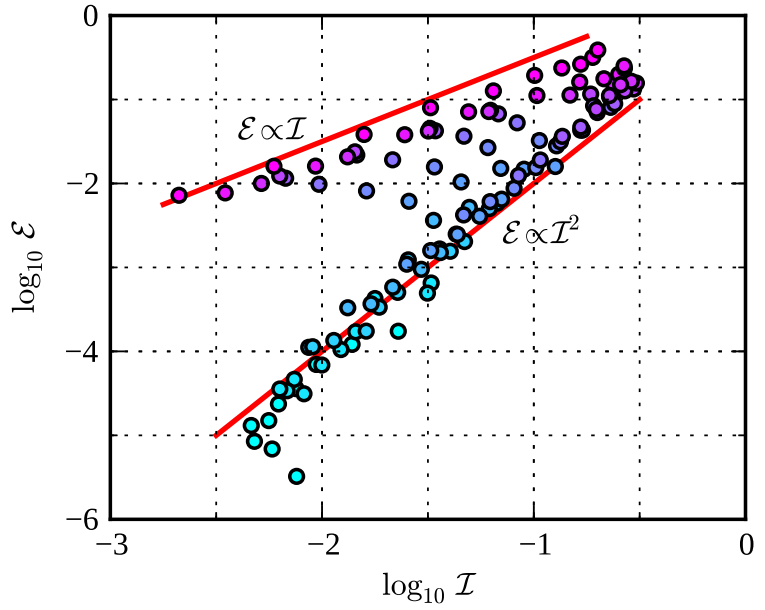

FIG. 5. The gamma-ray generation efficiency as function of the overall ion energy normalized to the initial energy of the laser pulse. The circles correspond to the results of numerical simulations used for Fig. 2. The colour corresponds to the laser intensity, from (cyan) $2 \times 10^{21} \mathrm{~W} \mathrm{~cm}^{-2}$ to (magenta) $2 \times 10^{25} \mathrm{~W} \mathrm{~cm}^{-2}$.

Despite ions on the front side of the foil are irradiated, the direct acceleration by the laser pulse is weaker than the acceleration by the induced plasma fields and can be neglected 31 . The threshold $n_{0}=\hat{n}_{0}$ is shown in Figs. 2 and 4 as line 3 .

Summarizing, on the (intensity, density) plane the region characterized by quick ion acceleration up to relativistic velocities is bound between lines corresponding to Eqs. (15) and (17) (lines 2 and 3 in Figs. 2 and (4). Surprisingly, this region approximately coincides with the area of efficient gamma-ray generation, as seen from Figs. 22 and 4. Besides, plasma dynamics is diverse enough in this region, e.g., it is quite irregular if $S>2 / l$ and regular if $S<2 / l$, at high intensities it is significantly affected by abundant positron production, etc. The considered coincidence can be partially explained by the proximity of the threshold intensity value for fast ion acceleration $\left(a_{0, i}=1 / 4 \pi\right.$, that yields $I \sim 10^{23} \mathrm{~W} \mathrm{~cm}^{-2}$ ) and the threshold intensity value for the radiation-dominated regime. Furthermore, two distinctive regimes with an evident relation between ion and gamma-ray energies are revealed by the results of the numerical simulations (see Fig. 5). Independently on the plasma density, at low laser intensity the final gammaray energy scales as the squared overall ion energy, and at high intensity the gamma-ray energy is in direct ratio with the ion energy. Thus, the mutual influence of gamma-ray generation and ion acceleration requires additional investigations.
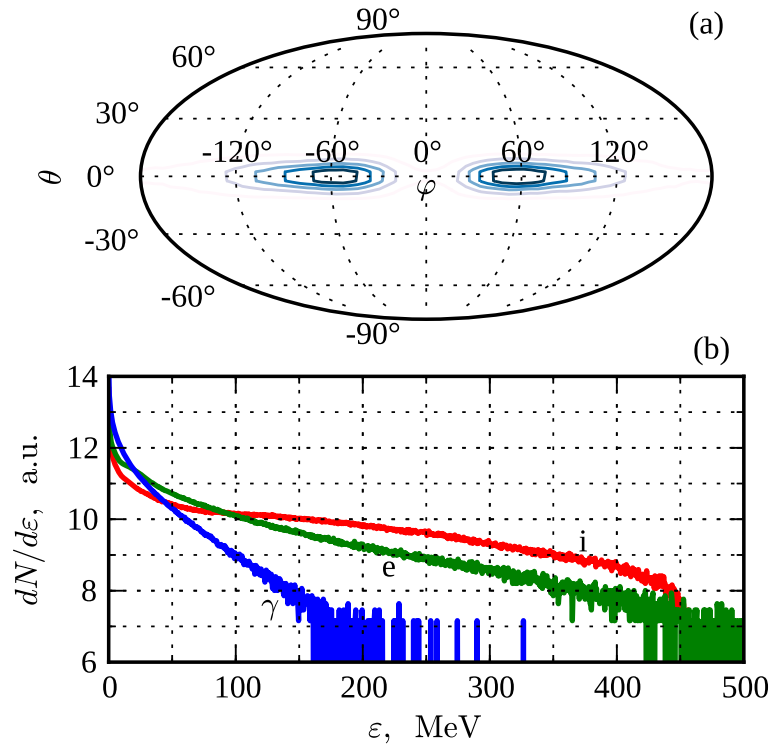

FIG. 6. (a) The Mollweide projection of the gamma-ray radiation pattern and (b) the photon, electron and ion spectra obtained in the numerical simulation corresponding to the point "A" in Fig. 11 The longitude $\varphi$ counts in the polarization plane, and the latitude $\theta$ counts from the polarization plane so that point $\varphi=0, \theta=0$ corresponds to the initial propagation direction of the laser pulse.

\section{SUMMARY AND DISCUSSION}

In conclusion, incoherent generation of hard photons in normal incidence of a laser pulse on a foil is considered by means of three-dimensional PIC $+\mathrm{MC}$ simulations. For various plasma densities and laser intensities the gammaray gain, directivity, generation efficiency and spectral features are found. The influence of ion dynamics on the emission of gamma-rays and the influence of radiation losses on the electron dynamics are discussed.

As seen from Fig. 1, the maximal gain at the intensity $3.2 \times 10^{23} \mathrm{~W} \mathrm{~cm}^{-2}\left(a_{0}=310\right)$ can be obtained if foil with $n_{e}=1.1 \times 10^{23} \mathrm{~cm}^{-3}\left(n_{0}=85\right)$ is used (see point "A" in Fig. (1). This intensity level is potentially attainable in the nearest future, so, let us consider in detail some properties of the gamma-ray bunches produced at this point. Thus, the generation efficiency is $9 \%$, the directivity is $\mathcal{D} \approx 12$, hence, the gain is about unity in this case. It should be noted that the generation efficiency drops dramatically at lower intensities and saturates at higher intensities, as seen from Fig. 3. The Mollweide projection of the gamma-ray radiation pattern and the photon spectrum are shown in Fig. [6] Two-lobe radiation pattern with lobes confined to the polarization plane is typical for the case of normal incidence $\stackrel{14,17}{ }$. One-lobe radiation pattern directed towards the incident laser pulse analogous to that in Ref. 15 reveals itself only at quite low intensities when the generation efficiency is not high.

The gamma-ray source corresponding to the point 
"A" in Fig. 1 for $1 \mathrm{~Hz}$ laser shot frequency produces every second $5 \times 10^{13}$ photons with energy greater than $0.5 \mathrm{MeV}$. For the photon energy of $1 \mathrm{MeV}$ such a source provides the spectral power of $2 \times$ $10^{10}$ photons $/ \mathrm{s} / 0.1 \%$ bandwidth and the spectral intensity of $2 \times 10^{4}$ photons $/ \mathrm{mrad}^{2} / \mathrm{s} / 0.1 \%$ bandwidth. Now let us compare these parameters with the parameters of modern Compton gamma-ray sources and discuss the potential applications of such a laser-plasma synchrotron source.

One of the most intense gamma-ray sources, $\mathrm{Hi} \gamma \mathrm{S}^{3}$, in a high-flux mode can produce about $10^{9}$ photons/s with average energy of $1 \mathrm{MeV}$. A collimator that provides $3 \%$ photon energy spread reduces the flux by the order of magnitude. Hence, $\mathrm{HI} \gamma \mathrm{S}$ on the level of $1 \mathrm{MeV}$ provides the spectral power of $3 \times 10^{6}$ photons $/ \mathrm{s} / 0.1 \%$ bandwidth that is four orders of magnitude lower than that for the considered laser-plasma source. However, HI $\gamma \mathrm{S}$ 's gamma-ray beams after the collimator spread by the angle of about $20 \mathrm{mrad}$ that yields the spectral intensity of $10^{4}$ photons $/ \mathrm{mrad}^{2} / \mathrm{s} / 0.1 \%$ bandwidth comparable with the considered all-optical gamma-ray source.

Another Compton source, produced quasimonoenergetic photon beams with the energy ranging from 0.1 to $0.9 \mathrm{MeV}$, has been recently developed ${ }^{4}$ at LLNL and used to perform nuclear resonance fluorescence (NRF) experiments ${ }^{5}$. This source yields $10^{5}$ photons/s with average energy corresponding to ${ }^{7} \mathrm{Li}$ line $(478 \mathrm{keV})$ and energy spread of $12 \%$. The resulting spectral power of $10^{3}$ photons/s/0.1\% bandwidth is enough for the accurate detection of ${ }^{7} \mathrm{Li}$ isotope in situ, however this required 7.5 hours of the operation ${ }^{5}$. The divergence of this gamma-ray beam is about $10 \mathrm{mrad}$, hence, not only the spectral power, but also the spectral intensity of the LLNL source is much lower than that for the considered laser-plasma source.

Despite of high potential performance of the laserplasma gamma-ray sources, some properties of them are so unfamiliar for nowadays gamma-ray physics that more sophisticated NRF experimental techniques can be required. For instance, the inherently wide photon spectrum will lead to extremely high Compton background in spectral measurements of re-emitted photons. Moreover, semiconductor and scintillating detectors generally used for gamma-ray energy measurements can operate only at the flux less than one photon per nanosecond. Thus, the NRF experiments will require detector arrays and a lot of the laser shots in order to obtain reasonable statistics. Nevertheless, femtosecond duration of gamma-ray bunches from laser-irradiated plasmas together with relatively long lifetimes of low-laying nuclear levels (generally from tens of femtoseconds to picoseconds ${ }^{32}$ ) might enable the time-of-flight separation ${ }^{33}$ of the Compton and NRF signals if an appropriate experimental design is proposed.

Obviously, gamma-ray beams from laser-plasma sources can be used in a number of applications that do not require high spectral quality. One of such an application is the radiography of ultrahigh density matter. It can be performed by means of laser-plasma sources with unprecedented time resolution, that is crucial for fast ignition experiments 34 . Another promising application of laser-plasma gamma-ray sources is a high-flux positron source based on pair creation by high-energy photons in a material target. Proposals of International Linear Collider suppose not only high electron and positron energy, but also high luminosity, that requires a reinforcement of nowadays positron sources. Relatively efficient positron production using Compton gamma-ray sources now is proved in the experiments, where gammato-positron conversion efficiency of about $10^{-3}$ and total positron flux of $10^{4} / \mathrm{s}$ are achieved ${ }^{35}$. Since the conversion efficiency does not sharply depend on the gammaray energy, the average flux of about $10^{10}$ positrons/s can be expected if the considered laser-plasma gammaray source is used.

Keeping on speculating about possible future experiments with bright gamma-ray beams, propagation-based polychromatic phase-contrast imaging 36 can be proposed. According to van Cittert-Zernike theorem the scale of the spatial coherence of light emitted by an incoherent source is about the product of light wavelength, distance to the source and reverse size of the source. Hence, the scale of the spatial coherence of a laser-plasma gamma-ray source is about $10 \mu \mathrm{m}$, if the photon energy is $1 \mathrm{MeV}$, the source size is $1 \mu \mathrm{m}$ and the distance from the source is $10 \mathrm{~m}$. Assuming that 100 gammaphotons belong to the corresponding solid angle, at least $10^{8}$ photons $/ \mathrm{mrad}^{2}$ are required for the phase-contrast gamma-imaging, that nearly the value of the considered source. Matter refractive index drops rapidly with the decrease of a hard-photon wavelength, however, the refractive index of polarized vacuum does not depend on the wavelength 28 . Hence, for a photon beam propagating through polarized vacuum the induced curvature of the beam phase front is higher for smaller wavelengths. This fact along with femtosecond duration of gammaray bunches from laser-irradiated plasmas can make such bunches a promising probe for the experiments on vacuum polarization in ultraintense laser field proposed in Ref. 37 .

Finally let us note that modern high-power laser facilities are already appropriate for quite efficient generation of high-energy photons in laser-plasma interactions. Simulation results of oblique incidence (the incidence angle is $60^{\circ}$ ) of a tightly focused $3 \mathrm{PW} p$-polarised laser pulse (FWHM: $3 \mu \mathrm{m}, 10 \mathrm{fs} ; \lambda=0.91 \mu \mathrm{m}, a_{0}=$ 100 , the peak intensity is $3.3 \times 10^{22} \mathrm{~W} \mathrm{~cm}^{-2}$ ) on a foil $\left(n_{e}=1.7 \times 10^{23} \mathrm{~cm}^{-3}, M / Z=2, l=1 \lambda\right)$ demonstrates reasonable generation efficiency $(0.7 \%)$ and unexpectedly very high directivity $(\mathcal{D}=37)$. The radiation pattern in this case consist of only one lobe lying both in the plane of incidence and in the foil plane, hence, the angle between the initial propagation direction of the laser pulse and the lobe direction is $30^{\circ}$. Under the assumption of $1 \mathrm{~Hz}$ laser system the resulting total gamma-ray flux is $10^{12}$ photons/s, the spectral power is 
$10^{8}$ photons $/ \mathrm{s} / 0.1 \%$ bandwidth and the spectral intensity is 400 photons $/ \mathrm{mrad}^{2} / \mathrm{s} / 0.1 \%$ bandwidth at the energy level of $1 \mathrm{MeV}$. Thus, quite high flux, spectral power and directivity of such a source can make it desirable for a number of applications and experiments.

Summarizing, theoretical analysis and threedimensional PIC+MC simulations demonstrate an interplay of ion acceleration and hard photon generation in laser-foil interactions. The scaling of the gamma-ray bunch parameters with the laser intensity is discussed. Properties and possible applications of the resulting gamma-ray bunches are considered, including nuclear resonance fluorescence and high-flux positron sources.

\section{ACKNOWLEDGMENTS}

This work has been supported in part by the Government of the Russian Federation (Project No. 14.B25.31.0008) and by the Russian Foundation for Basic Research (Grants No 13-02-00886, 13-02-97025).

${ }^{1}$ A. Wagner, R. Beyer, F. Erhard, M. Dnau, E. Grosse, A. Hartmann, A. R. Junghans, L. Kubler, K. Kosev, S. Mallion, C. Nair, N. Nankov, G. Rusev, K. D. Schilling, W. Schulze, and R. Schwengner, Journal of Physics G: Nuclear and Particle Physics 31, S1969 (2005) ${ }^{2}$ D. H. Bilderback, P. Elleaume, and E. Weckert, Journal of Physics B: Atomic, Molecular, and Optical Physics 38, ${ }^{3}$ H. R. Weller, M. W. Ahmed, H. Gao, W. Tornow, Y. K. $\mathrm{Wu}, \mathrm{M}$. Gai, and R. Miskimen, Progress in Particle and Nuclear Physics 62, 257 (2009).

${ }^{4}$ D. J. Gibson, F. Albert, S. G. Anderson, S. M. Betts, M. J. Messerly, H. H. Phan, V. A. Semenov, M. Y. Shverdin, A. M. Tremaine, F. V. Hartemann, C. W. Siders, D. P. McNabb, and C. P. J. Barty, Physical Review Special Topics - Accelerators and Beams 13, 70 ${ }^{5}$ F. Albert, S. G. Anderson, D. J. Gibson, C. A. Hagmann, M. S. Johnson, M. Messerly, V. Semenov, M. Y. Shverdin, B. Rusnak, A. M. Tremaine, F. V. Hartemann, C. W. Siders, D. P. McNabb, and C. P. J. Barty, Physical Review Special Topics - Accelerators and Beams 13, 707
${ }^{6}$ K. Ta Phuoc, S. Corde, C. Thaury, V. Malka, A. Tafzi, J. P. Goddet, R. C. Shah, S. Sebban, and A. Rousse, Nature Photonics 6, 308 (2012) N. D. Powers, I. Ghebregziabher, G. Golovin, C. Liu, S. Chen, S. Banerjee, J. Zhang, and D. P. Umstadter, Nature Photonics 8, 28 (2014)

${ }^{7}$ E. Nerush and I. Kostyukov, Physical Review E 75, 057401 (2007)

${ }^{8}$ J. D. Kmetec, C. L. Gordon, J. J. Macklin, B. E. Lemoff, G. S. Brown, and S. E. Harris, Physical Review Letters 68, 1527 (1992) P. A. Norreys, M. Santala, E. Clark, M. Zepf, I. Watts, F. N. Beg, K. Krushelnick, M. Tatarakis, A. E. Dangor, X. Fang, P. Graham, T. McCanny, R. P. Singhal, K. W. D. Ledingham, A. Creswell, D. C. W. Sanderson, J. Magill, A. Machacek, J. S. Wark, R. Allott, B. Kennedy, and D. Neely, Physics of Plasmas 6, 2150 (1999) S. P. Hatchett, C. G. Brown, T. E. Cowan, E. A. Henry, J. S. Johnson, M. H. Key, J. A. Koch, A. B. Langdon, B. F. Lasinski, R. W. Lee, A. J. MacKinnon, D. M. Pennington, M. D. Perry, T. W. Phillips, M. Roth, T. C. Sangster, M. S. Singh, R. A. Snavely, M. A. Stoyer, S. C. Wilks, and K. Yasuike, Physics of Plasmas 7, 2076 (2000)

${ }^{9}$ H. Chen, S. C. Wilks, J. D. Bonlie, E. P. Liang, J. Myatt, D. F. Price, D. D. Meyerhofer, and P. Beiersdorfer, Physical Review Letters 102, 105001 (2009)
${ }^{10}$ A. I. Nikishov and V. I. Ritus, Sov. Phys. JETP 19, 529 (1964); V. I. Ritus, Journal of Russian Laser Research 6, 497 (1985)

${ }^{11}$ S. V. Bulanov, T. Z. Esirkepov, J. Koga, and T. Tajima, Plasma Physics Reports 30, 196213 (2004); E. Esarey, S. K. Ride, and P. Sprangle, Phys. Rev. E 48, 3003 (1993)

${ }^{12}$ V. Yanovsky, V. Chvykov, G. Kalinchenko, P. Rousseau, T. Planchon, T. Matsuoka, A. Maksimchuk, J. Nees, G. Cheriaux, G. Mourou, and K. Krushelnick, Optics Express 16, 2109 (2008)

${ }^{13}$ G. A. Mourou, T. Tajima, and S. V. Bulanov, Rev. Mod. Phys. 78, 309 (2006) A. Di Piazza, C. Mller, K. Z. Hatsagortsyan, and C. H. Keitel, Rev. Mod. Phys. 84, 1177 (2012)

${ }^{14}$ T. Nakamura, J. K. Koga, T. Z. Esirkepov, M. Kando, G. Korn, and S. V. Bulanov, Phys. Rev. Lett. 108, 195001 (2012)

${ }^{15}$ C. S. Brady, C. P. Ridgers, T. D. Arber, A. R. Bell, and J. G. Kirk, Phys. Rev. Lett. 109, 245006 (2012)

${ }^{16} \mathrm{D}$. An der Brgge and A. Pukhov, Physics of Plasmas 17, 3110 (2010) A. A. Gonoskov, A. V. Korzhimanov, A. V. Kim, M. Marklund, and A. M. Sergeev, Physical Review E 84, 046403 (2011) J. Sanz, A. Debayle, and K. Mima, Physical Review E 85, 46411 (2012) S. V. Bulanov, T. Z. Esirkepov, M. Kando, S. S. Bulanov, S. G. Rykovanov, and F. Pegoraro, Physics of Plasmas 20, 3114 (2013)

${ }^{17}$ C. P. Ridgers, C. S. Brady, R. Duclous, J. G. Kirk, K. Bennett, T. D. Arber, A. P. L. Robinson, and A. R. Bell, Phys. Rev. Lett. 108, 165006 (2012)

${ }_{18}$ A. Bell and J. Kirk, Physical Review Letters 101, 200403 (2008)

${ }^{19}$ A. M. Fedotov, N. B. Narozhny, G. Mourou, and G. Korn, Phys. Rev. Lett. 105, 080402 (2010)

${ }^{20}$ C. P. Ridgers, C. S. Brady, R. Duclous, J. G. Kirk, K. Bennett, T. D. Arber, and A. R. Bell, Physirs of Plasmas 20,6701 (2013)

7781 (2). N. V. Elkina, and H. Ruhl, Phys. Rev. Lett. 106, 035001 (2011)

${ }^{22}$ E. N. Nerush and I. Y. Kostyukov, Nuclear Instruments and Methods in Physics Research A 653, 7 (2011)

${ }^{23}$ A. Pukhov, J. Plasma Physics 61, 425433 (1999).

${ }^{24}$ J.-L. Vay, Physics of Plasmas 15, 056701 (2008)

${ }^{25}$ A. A. Soloviev, M. V. Starodubtsev, K. F. Burdonov, I. Y. Knstyukov, E. N. Nerush, A. A. Shaykin, and E. A. Khazanov, (2hididiew of Scientific Instruments 82, 3304 (2011)

${ }^{26}$ N. V. Elkina, A. M. Fedotov, I. Y. Kostyukov, M. V. Legkov, N. B. Narozhny, E. N. Nerush, and H. Ruhl, Physical Review Special Topics - Accelerators and Beams 14, 54401 (2011)

$27 \mathrm{~V}$ N. Baier and V. M. Katkov, Physics Letters A 25, 492 (1967) (2Q10X. Baier, V. Katkov, and V. Strakhovenko, Electromagnetic processes at high energies in oriented single crystals (World Scientific, Singapore, 1998).

${ }^{28}$ V. B. Berestetskii, E. M. Lifshitz, and L. P. Pitaevskii, Quantum Electrodynamics (Pergamon, New York, 1982).

${ }^{29} \mathrm{~S}$ Gordienko and A. Pukhov, Physics of Plasmas 12, 3109 (2005)

${ }^{30}$ L. D. Landau and E. M. Lifshitz, edited by T. C. T. of Fields (Elsevier, Oxford, 1975).

${ }^{31}$ T. Esirkepov, M. Borghesi, S. V. Bulanov, G. Mourou, and T. Tajima, Phys. Rev. Lett. 92, 175003 (2004).

${ }^{32}$ M. B. Chadwick, P. Obloinsk, M. Herman, N. M. Greene, R. D. McKnight, D. L. Smith, P. G. Young, R. E. Macfarlane, G. M. Hale, S. C. Frankle, A. C. Kahler, T. Kawano, R. C. Little, D. G. Madland, P. Moller, R. D. Mosteller, P. R. Page, P. Talou, H. Trellue, M. C. White, W. B. Wilson, R. Arcilla, C. L. Dunford, S. F. Mughabghab, B. Pritychenko, D. Rochman, A. A. Sonzogni, C. R. Lubitz, T. H. Trumbull, J. P. Weinman, D. A. Brown, D. E. Cullen, D. P. Heinrichs, D. P. McNabb, H. Derrien, M. E. Dunn, N. M. Larson, L. C. Leal, A. D. Carlson, R. C. Block, J. B. Briggs, E. T. Cheng, H. C. Huria, M. L. Zerkle, K. S. Kozier, A. Courcelle, V. Pronyaev, and S. C. van der Marck, Nuclear Data Sheets 107, 2931 (2006).

${ }^{33}$ M. W. Ahmed, G. Feldman, V. N. Litvinenko, S. O. 
Nelson, B. E. Norum, B. Perdue, I. V. Pinayev, B. Sawatzky, A. P. Tonchev, Y. Wu, and H. R. Weller, lier, Nuclear Fusion 44, 266 (2004) Nuclear Instruments and Methods in Physics Research Section A 516, R40KQ004t), M Nomura, A, Ohashi, T, Okugi, K. Sakau ${ }^{34}$ C. P. J. Barty, M. Key, J. Britten, R. Beach, G. Beer, C. Brown, S. Bryan, J. Caird, T. Carlson, J. Crane, J. Dawson, A. C. Erlandson, D. Fittinghoff, M. Hermann, C. Hoaglan, A. Iyer, I. Jones, L., I. Jovanovic, A. Komashko, O. Landen, Z. Liao, W. Molander, S. Mitchell, E. Moses, N. Nielsen, H.-H. Nguyen, J. Nissen, S. Payne, D. Pennington, L. Risinger, M. Rushford, K. Skulina, M. Spaeth, B. Stuart, G. Tietbohl, and B. WattelT. Saito, J. Urakawa, M. Washio, Physical Review Letters 96, 114801 (2006)

${ }^{36}$ S. W. Wilkins, T. E. Gureyev, D. Gao, A. Pogany, and A. W. Stevenson, Nature 384, 335 (1996)

${ }^{37}$ B. King, A. di Piazza, and C. H. Keitel, Nature Photonics 4, $92(2010) \quad$ B. King, A. Di Piazza, and C. H. Keitel, Phys. Rev. A 82, 032114 (2010) 